\title{
The Optimization of the Search Scheme by the Simulated Annealing Algorithm
}

\author{
Guangshuo Niu \\ North China Electric Power University, Baoding 071000, China \\ 1150995425@qq.com
}

Keywords: Optimization; Search Scheme; Simulated Annealing Algorithm

\begin{abstract}
Since the airplane is invented by Wright Brothers, it has greatly improved people's traffic efficiency and produced large quantity of fortunes. We built a model to draw a sensible searching scheme.

The major assumptions are: 1) the wind and the ocean current are only related to the geography location and the season, 2) the velocity of the ocean current is linearly reduced according to the depth under the seawater, 3) the aircraft wreck will be kept stable when it arrives at the seabed.

We analyze the plane wreck's motion in the horizontal direction and the vertical direction, respectively, taking the ocean current, the wind force, and the Coriolis Effect into account at the same time. Thirdly, we use the clustering algorithm of DBSCAN to calculate the focused searching areas. Finally, we adopt the simulated annealing algorithm to arrange the search aircrafts and optimize the searching routes for scanning the focused areas.
\end{abstract}

\section{Introduction}

Now the flight has become an indispensable transport mode. However, the frequent crash accidents occurred recently have greatly scared many people. The key problem to maximally save people's life after the crash is how to successfully search and find the lost plane as quickly as possible. It is also significant to rebuild people's confidence and enthusiasm on taking a flight travel.

By far, though the international community has established the safety system and studied the offshore drifting models, the more proper method for the searching work, especially the way to distinguish the probability distribution of the location, is still need to be developed.

\section{The searching orders of focused areas}

These five major areas should be searched in a certain period of time with high efficiency. So it is needed for us to make a reasonable calculation to optimize the search route. We use the simulated annealing algorithm to calculate the shortest path for the search. And the main principles are as follows.

1. We use $d_{i, j}$ to represent the distance between point $i$ and point $j . \pi_{i}=j$ is the search goal for the $(i-1)^{\text {th }}$ time. And the objective function is

$$
\min f\left(\pi_{1} \pi_{2} \cdots \pi_{k}\right)
$$

2. After exchanging the order of $u$ and $v$, we get the new route as follows

$$
\pi_{1} \cdots \pi_{u-1} \pi_{v} \pi_{v-1} \cdots \pi_{u+1} \pi_{u} \pi_{v+1} \pi_{102}
$$

3. According to the above exchange, the difference of the routes is

$$
\Delta f=\left(d_{\pi_{u-1 \pi_{v}}}+d_{\pi_{u} \pi_{v+1}}\right)-\left(d_{\pi_{u-1 \pi_{u}}}+d_{\pi_{v} \pi_{v+1}}\right)
$$

4. Setting a new route rule via

$$
p= \begin{cases}1 & \Delta f<0 \\ e^{\left(-\frac{\Delta f}{T}\right)} & \Delta f \geq 0\end{cases}
$$


We choose the new route when $\Delta f<0$. Otherwise, we use $e^{\left(-\frac{\Delta f}{T}\right)}$ as a new route.

5. We compute the cooling coefficient with $\alpha=0.999$ through

$$
T \longleftarrow \alpha T
$$

6. We judge that whether the annealing algorithm is finished by the end temperature $e=10^{-30}$. When $T<e$, the algorithm is finish and we get the current state.

Based on the simulated annealing algorithm, we calculate the best searching route as shown in Figure 1.

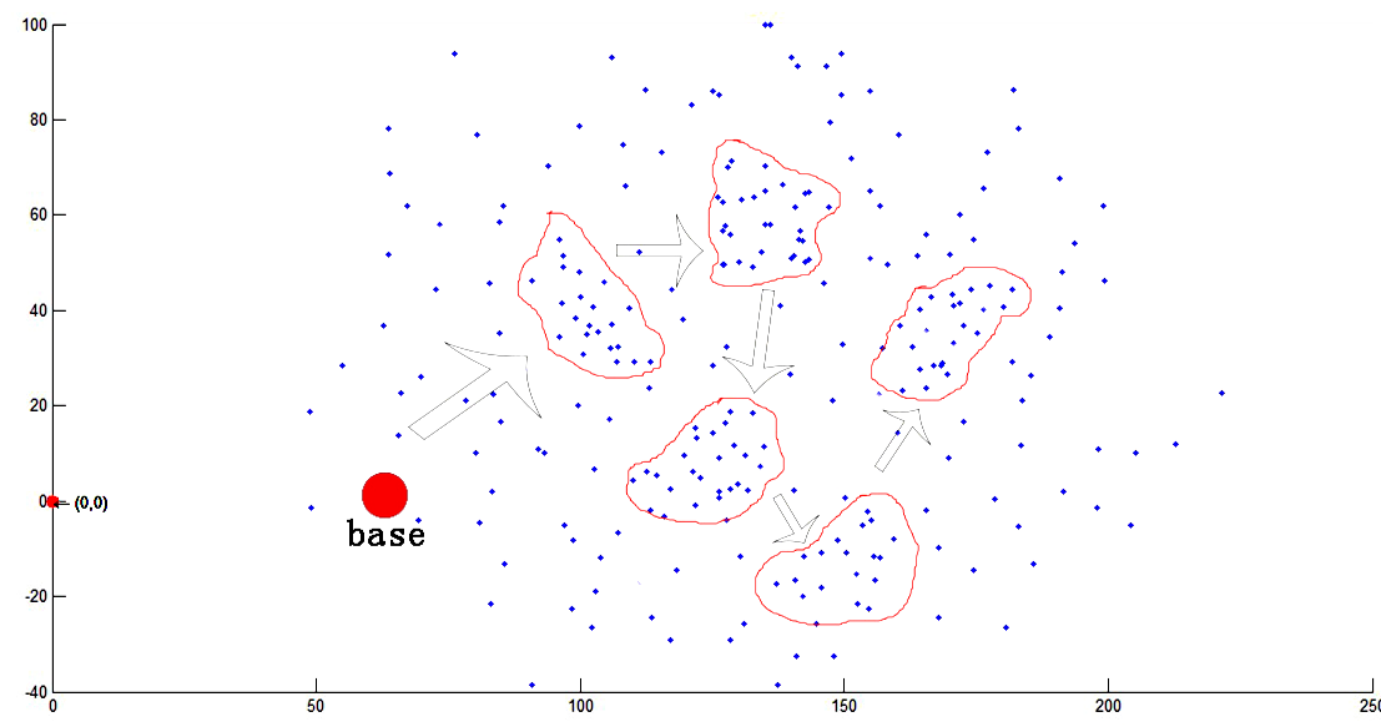

Figure 1. The best searching routes

\section{Searching arrangement of the focused areas}

We determine the wreck lies equiprobable in each searching area if there is no information indica ting which area is more likely to have the plane wreck [1]. In our paper, considering that different types of searching aircrafts and ships have different rescue abilities, we enhance the searching efficiency and reduce the searching time.

According to the real time probability of the wreck's location, we search the major areas including the horizontal search and the vertical search. Finally we obtain the 3D probability distribution of the searching domain, as shown in Figure 2.

We search the area whose length is $\boldsymbol{a}$ and width is $\boldsymbol{b}$. Considering the different searching ability of aircrafts, we assume that there are $m$ aircrafts. And there are $i$ aircrafts who can search to the depth of $h_{i}$ and areas of $s_{i}$. Generally, the larger $h_{i}$ is, the bigger $s_{i}$ will be. To complete the searching as soon as possible, we should send all of the available aircrafts[2]. The usual searching route is as Figure 3. 


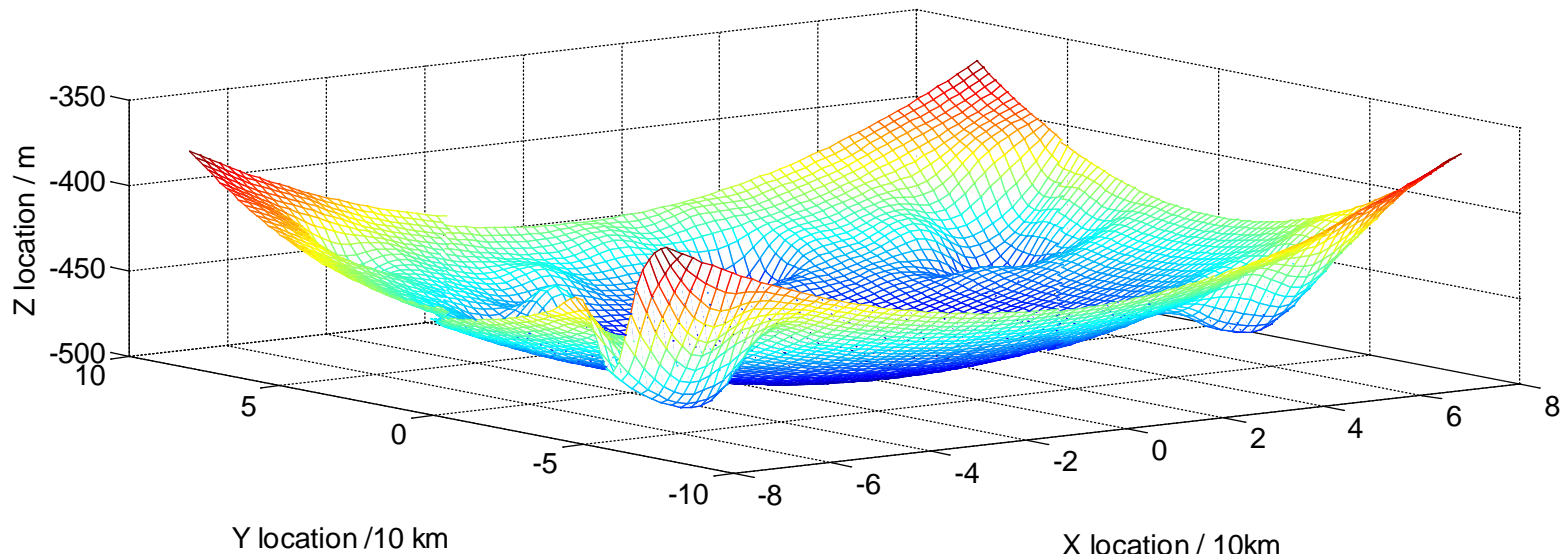

Figure 2.The 3D probability distribution of the searching domain

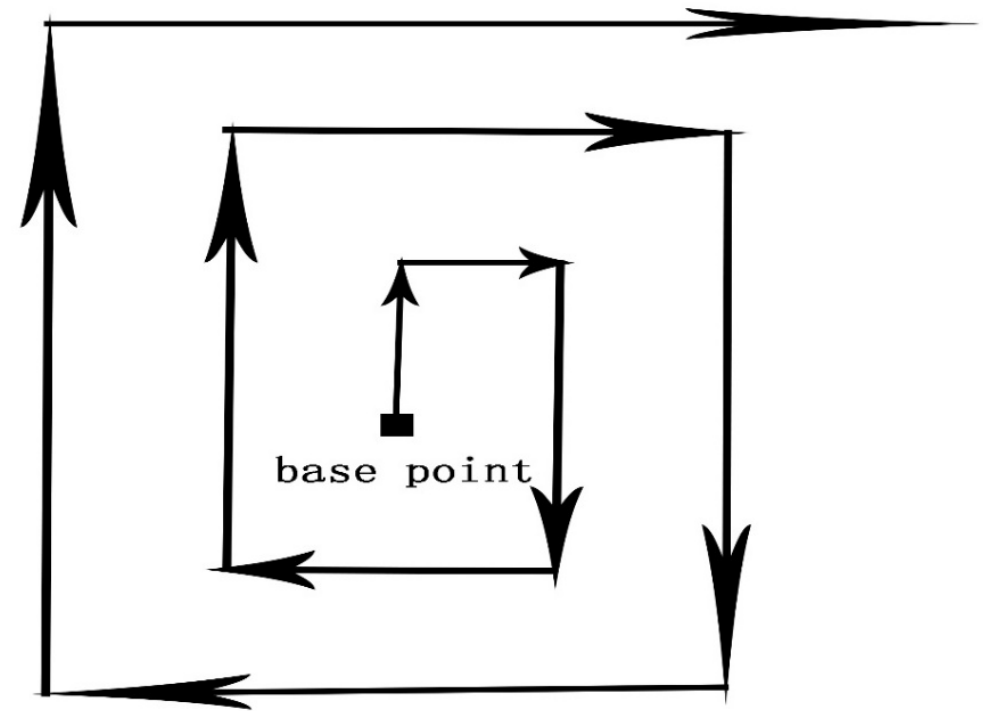

Figure 3. Searching line

We divide the searching areas into $m$ rectangles, and the $j^{\text {th }}$ rectangle's length, width and depth are $X_{j}, Y_{j}$ and $Z_{j}$, respectively. There are $i$ aircrafts searching $j$ areas, then we have

And the objective function is

$$
\left\{\begin{array}{l}
t S_{i} \geq X_{i} Y_{j} \\
h_{i} \geq z_{j} \\
\sum\left(X_{i} Y_{j}\right)=a b \\
\sum X_{i} \geq a \\
\sum Y_{i} \geq b \\
\left\{s_{j}\right\}=S
\end{array}\right.
$$

$$
f=\min (t)
$$

We get the division of the area $\boldsymbol{S}$ through our scheme as follows 


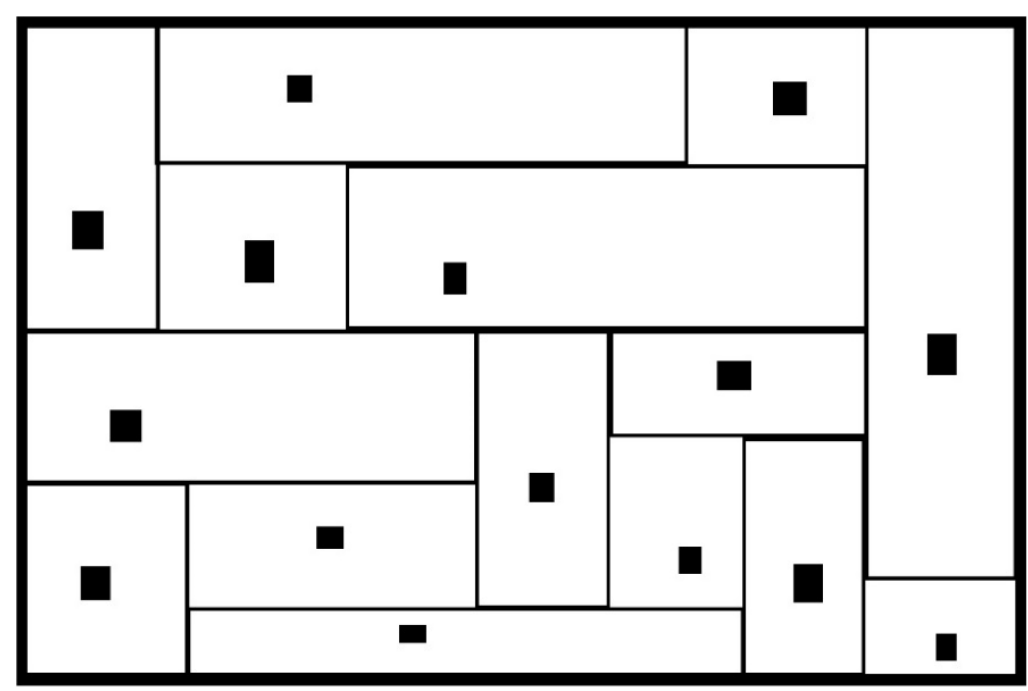

the deepest place in every area

Figure 4. The divide of searching areas

\section{The verification result of our model}

We apply our model to make a simulation on the practical lost plane of Air France in 2009 and find that, 1) the final location of the plane wreck obtained by the simulation is very close to the practical one which has been confirmed, and 2) it only takes 18 days, which is much less than the practical time of two years, for our model to find the lost plane. So our model is effective and precise.

\section{References}

[1] Sheng-wei Xing, Research on Global Optimization Model and Simulation of Joint Aeronautical and Maritime Search, Dalian Maritime University for the degree of Doctor of Engineering, September 2012.

[2] Shao-rong Feng, Wen-jun Xiao, An Improved DBSCAN Clustering Algorithm, Journal of China University of Mining\& Technology, Vol.37 No.1, Jan. 2008 